\title{
Intracoronary administration of levosimendan in patients with acute coronary syndromes and decreased left ventricular ejection fraction undergoing coronary artery bypass graft surgery
}

\author{
VJERA MARINOV ${ }^{1}$, NENAD KARANOVIC ${ }^{2}$, MLADEN CAREV ${ }^{2}$, CRISTIJAN BULAT ${ }^{3}$, DUBRAVKA KO- \\ CEN $^{2}$, MIHAJLO LOJPUR ${ }^{2}$, ZDENKO COVIC $^{2}$, BOZENA IVANCEV $^{2}$, ZVONIMIR PARCINA $^{2}$
}

${ }^{1}$ Anesthesiology Unit, Cito Clinic Split

${ }^{2}$ Department of Anesthesiology, Reanimatology and Intensive Care, University Hospital Split

${ }^{3}$ Department of Cardiac Surgery, University Hospital Split

Corresponding author:

Mladen Carev

Department of Anesthesiology, Reanimatology and Intensive Care

University Hospital Split

Spinciceva 1, 21000 Split, Croatia

Phone: +38521556180

Fax: +38521556580

E-mail:mladen.carev1@gmail.com

\section{ABSTRACT}

In cardiac surgery patients, intracoronary (IC) administration of levosimendan can provide optimal drug spread, enabling effective manifestation of favorable drug effects and avoiding potentially harmful systemic hypotension. This could be beneficial in acute coronary syndromes (ACS) with decreased left ventricular ejection fraction (LVEF). We present ten cases of IC administration of levosimendan in ACS manifested as ST segment elevation myocardial infarction, non-ST segment elevation myocardial infarction or unstable angina pectoris. All patients underwent coronary artery bypass graft (CABG) surgery, performed as an "off-pump" or "onpump"/“off-clamp" procedure (latter one with the use of cardiopulmonary bypass on the beating heart). Levosimendan was administered as an IC bolus $(125-250 \mu \mathrm{g})$ in each coronary artery graft (2-3 grafts). Intravenous (IV) levosimendan infusion continued $(0.1-0.2 \mu \mathrm{g} \bullet \mathrm{kg}-1 \bullet \mathrm{min}-1)$ after graft placements (24-48 h), with IV infusion of norepinephrine $(0.1 \mathrm{mg} \bullet \mathrm{ml}-1)$, if needed. Cardiac function was assessed using LVEF (\%) (Teicholz), thermodilution cardiac in$\operatorname{dex}(\mathrm{CI})(\mathrm{ml} \bullet \mathrm{m}-2)$, and systemic vascular resistance (SVR) (dynes•sec $\bullet \mathrm{cm}-5)$.

Nonparametric Wilcoxon signed-ranks test [presented as median (MED) with interquartile range (IQR)] indicated a significant difference between preoperative vs. immediate postoperative CI, SVR, and LVEF in all cases [2.2 (1.9-2.5) vs. 3.1 (2.9-
3.4) $\mathrm{ml} \bullet \mathrm{m}-2,1173.0(1062.7-1278.2)$ vs. 882.5 (763.5-993.0) dynes•sec•cm-5, 44.5 (36.0-46.7) vs. 53.5 (45.7-59.2) \%, respectively] $(\mathrm{P}=0.005)$, i.e. IC administration of levosimendan was associated with prompt improvement of intraoperative hemodynamics and cardiac contractility. IC administration of levosimendan may be a promising alternative method for improving decreased cardiac function in acute cardiac ischemia, besides necessary surgical revascularization.

Key words: levosimendan, intracoronary, acute coronary syndromes, CABG surgery

\section{INTRODUCTION}

Patients with acute coronary syndrome (ACS), undergoing surgical myocardial revascularization, are susceptible to contractile myocardial dysfunction with hemodynamic compromise, often necessitating inotropic and/or mechanical circulatory support. $(1,2)$ Consequently, perioperative preservation of heart function and the use of optimal inotropic agents are becoming strongly emphasized. $(2,3)$ A calcium-sensitizing drug, levosimendan, has emerged as a novel inotropic agent with vasodilator properties and potent cardioprotective effects, all especially beneficial in coronary artery disease (CAD). $(2,4)$ The perioperative use of levosimendan in cardiac surgery patients has been associated with a reduction in myocardial injury, improvement of hemodynamics and clinical outcomes, and reduction in additional inotropic and/ or mechanical circulatory support. $(1,4)$ In the majority of studies, levosimendan is usually administered as a continuous intravenous (IV) infusion over $24 \mathrm{~h}$. (4) However, IV use of levosimendan could be limited by extensive systemic vasodilatation and hypotension, particularly in CAD. (5) Intracoronary (IC) administration of levosimendan was proposed as a possible strategy for effective regional myocardial drug distribution, facilitating favorable and avoiding potentially harmful drug effects. (6-9)

We present 10 cases of IC administration of levosimendan in ACS with decreased left ventricular ejection fraction (LVEF), manifested as ST segment elevation myocardial infarction (STEMI), non-ST segment elevation myocardial infarction (NSTEMI) or unstable angina pectoris (AP), requiring coronary artery bypass graft (CABG) surgery.

\section{MATERIALS AND METHODS}

The study was approved by the local Ethics Committee of the University Hospital Split.

\section{Technical aspects of IC levosimendan administration}

CABG surgery was performed as an "offpump" or "on-pump"/“off-clamp" proce- 
dure, the latter with the use of cardiopulmonary bypass (CPB) on the beating heart. Levosimendan was administered as an IC bolus injected in each coronary artery graft. Levosimendan was first diluted ( 12.5 $\mathrm{mg}$ in $50 \mathrm{ml}$ of $5 \%$ glucose solution, 250 $\mu \mathrm{g} \bullet \mathrm{ml}-1)$. Two $\mathrm{ml}$ of this drug solution was mixed with the patients heparinized blood in a $20 \mathrm{ml}$ syringe $(25 \mu \mathrm{g} \bullet \mathrm{ml}-1)$. Prepared content from the syringe was forwarded to the surgery team in sterile conditions. A surgeon administered 5-10 ml (125$250 \mu \mathrm{g})$ in each coronary artery graft $(2-3$ grafts). After the completion of CABG surgery, IV infusion of levosimendan continued (0.1-0.2 $\mu \mathrm{g} \bullet \mathrm{kg}-1 \bullet \mathrm{min}-1)$ to prolong drug delivery (24-48 h), with IV infusion of norepinephrine $(0.1 \mathrm{mg} \bullet \mathrm{ml}-1)$, if needed.

\section{Patients}

Our first case (45-year-old female in acute STEMI), was done unintentionally in an urgent manner, due to acute onset of myocardial stunning during weaning from $\mathrm{CPB}$. Surgical revascularization started as Off-pump coronary artery bypass (OP$\mathrm{CAB})$,, but after the first bypass was completed, the patient experienced sudden hemodynamic instability. An intra-aortic balloon pump (IABP) was positioned without expected improvement. Urgent $\mathrm{CPB}$ was initiated in order to place two additional grafts. During the very challenging process of weaning (hemodynamic compromise, several weaning attempts), we decided to administer an IC bolus of levosimendan in each coronary artery graft (in consultancy with colleagues from abroad). Soon after, the patient was successfully weaned from CPB with evident intraoperative improvement of hemodynamics and overall cardiac function. We continued with IC levosimendan administration in several subsequent acute STEMI cases with troponin I over $50 \mathrm{ng} \bullet \mathrm{ml}-1$ (5 cases). Additionally, as we continued, we found this method to be beneficial in more complicated cases of advanced chronic CAD manifested in acute form, as unstable AP ( 4 cases) or acute NSTEMI (1 case), with moderately elevated troponin I.

\section{Perioperative evaluation of cardiac function and outcomes}

Cardiac function was obtained with perioperative heart ultrasound estimation of LVEF (\%) (Teicholz), thermodilution cardiac index $(\mathrm{CI})(\mathrm{ml} \bullet \mathrm{m}-2)$, and systemic vascular resistance (SVR) (dynes॰sec $\bullet$ cm-5). Troponin I (ng•ml-1) was measured pre- operatively and checked regularly. Time to extubation, intensive care unit (ICU) and hospital discharge were expressed in postoperative days (POD). POD 1 represented the first day following the day of surgery.

\section{Statistical analysis}

The results were analyzed using statistical program SPSS 14.0 with nonparametric Wilcoxon signed-ranks test for comparisons of preoperative vs. immediate postoperative values (or ICU discharge values for troponin I) of CI, SVR, LVEF, and troponin I variables in all patients. Data were presented as median (MED) with interquartile range (IQR). Statistical significance was set at $\mathrm{P}<0.05$.

\section{RESULTS}

General characteristics, clinical findings and outcomes in each of ten patients are presented in table 1. Comparing preoperative vs. immediate postoperative $\mathrm{CI}$, SVR, and LVEF in all patients (table 2), IC administration of levosimendan exerted significant improvement of intraoperative hemodynamics and cardiac contractility $(\mathrm{P}=0.005)$. Preoperative vs. ICU discharge troponin I decreased in all cases $(\mathrm{P}=0.005)$ (table 2).

\section{DISCUSSION}

In this short report, IC administration of levosimendan was associated with evident improvement in intraoperative hemodynamics and overall cardiac function.

Jamali et al. have previously found that IC levosimendan administration enhances contractile function of stunned myocardium. (10) Several subsequent animal studies pointed to its cardioprotective and antiapoptotic effects. $(6,7)$ In humans, IC administration of levosimendan improved systolic and diastolic cardiac function, in ischemic and non-ischemic acute heart failure, without significant systemic hypotension. $(8,9)$ Our main reason for IC administration of levosimendan was globally hypokinetic myocardium with reduced overall cardiac function evident in every patient. Patients who underwent an "on pump"/"off-clamp" procedure were successfully weaned from CPB. In "off pump" cases, an IC bolus was first administered after the placement of the first graft, enabling hemodynamic stability and procedure continuation. In previous studies, IC levosimendan was used in variable doses, as an IC bolus or short continuous IC infusion. (7-9) An ongoing clinical trial chose a $12 \mu \mathrm{g} \bullet \mathrm{kg}-1$ dose of IC levosimendan infusion at the induction of cardioplegia (after opening of the aortic cross-clamp in aortic valve/CABG surgery). (4) Our 125-250 $\mu \mathrm{g}$ IC bolus administered in each coronary artery graft (2-3 grafts) during surgical revascularization, corresponded approximately to the $6-12 \mu \mathrm{g} \bullet \mathrm{kg}-1$ dose of IC levosimendan administered to each patient. Many questions arise regarding the right timing for IC levosimendan administration, optimal dose, category of patients that could benefit, and the relevant outcome measures. Further randomized control studies and/or more case series are needed to provide the correct answers.

In conclusion, as we used an IV infusion of levosimendan (following graft placements) and surgical revascularization, it is not possible to define the exact contribution of IC levosimendan to the final clinical outcome. However, we believe that IC administration of levosimendan may be a useful alternative method for improving decreased cardiac function in acute onset cardiac ischemia, in addition to necessary surgical revascularization.

\section{ACKNOWLEDGEMENTS}

The authors wish to thank Sladjana Malbasa, bacc. med. techn., Andjela Pastar, bacc. med. techn., Anita Radan, bacc. med. techn., Jasenka Kosor, med. techn., and Marija Mamic, bacc. med. techn., for their technical and professional support. 
Table 1. General characteristics, clinical findings and outcomes in patients with ACS administered IC levosimendan during CABG surgery.

\begin{tabular}{|c|c|c|c|c|c|c|c|c|}
\hline case & $\begin{array}{l}\text { gender/age } \\
\text { BMI }\end{array}$ & diagnosis & surgery & remarks & $\begin{array}{l}\text { levosimendan } \\
\text { IC bolus } \\
(125-250 \mu \mathrm{g})\end{array}$ & $\begin{array}{l}\text { cardiac function } \\
\text { preop. / postop. }\end{array}$ & $\begin{array}{l}\text { troponin I } \\
(\text { ng.ml-1) } \\
\text { preop. / ICU } \\
\text { discharge }\end{array}$ & outcomes \\
\hline 1 & $\begin{array}{l}\mathrm{F} / 45 \\
23.0\end{array}$ & STEMI & $\begin{array}{l}\text { CABG x } 3 \\
\text { IABP }\end{array}$ & $\begin{array}{l}\text { HD } 11 \text { years } \\
\text { kidney trans- } \\
\text { plantation }\end{array}$ & $\begin{array}{l}3 \text { boluses }+0.1-0.2 \mu \mathrm{g} \\
\mathrm{kg} / \mathrm{min}(48 \mathrm{~h})\end{array}$ & $\begin{array}{l}\text { /CI } 1.9 \text { / } 3.0 \\
\text { SVR } 1223 \text { / } 1000 \\
\text { LVEF 44\% / 53\% }\end{array}$ & $>50 / 7.044$ & $\begin{array}{l}\text { extubation - POD } 3 \\
\text { ICU discharge - POD } 5 \\
\text { hospital discharge - } \\
\text { POD } 18\end{array}$ \\
\hline 2 & $\begin{array}{l}M / 68 \\
27.4\end{array}$ & STEMI & $\begin{array}{l}\text { CABG } \times 3 \\
\text { IABP }\end{array}$ & $\begin{array}{l}\text { previous } 1 \mathrm{MI} \text {, } \\
\mathrm{AH}\end{array}$ & $\begin{array}{l}3 \text { boluses }+0.1-0.2 \mu \mathrm{g} \\
\mathrm{kg} / \mathrm{min}(36 \mathrm{~h})\end{array}$ & $\begin{array}{l}\text { /CI } 2.6 \text { / } 3.3 \\
\text { SVR } 1071 \text { / } 919 \\
\text { LVEF } 45 \% \text { / 57\% }\end{array}$ & $>50 / 19.442$ & $\begin{array}{l}\text { extubation - POD } 1 \\
\text { ICU discharge - POD } 3 \\
\text { hospital discharge - } \\
\text { POD } 9\end{array}$ \\
\hline 3 & $\begin{array}{l}\mathrm{F} / 44 \\
34.2\end{array}$ & STEMI & $\begin{array}{l}\text { CABG x } 3 \\
\text { IABP }\end{array}$ & $\begin{array}{l}\text { AH, CRRT } 2 \\
\text { days, } \\
\text { intrapleural } \\
\text { hemorrhage }\end{array}$ & $\begin{array}{l}3 \text { boluses }+0.15-0.2 \\
\mu \mathrm{g} / \mathrm{kg} / \mathrm{min}(24 \mathrm{~h})\end{array}$ & $\begin{array}{l}\text { CI } 2.7 \text { / } 3.8 \\
\text { SVR } 1237 \text { / } 810 \\
\text { LVEF 50\% / 60\% }\end{array}$ & $>50 / 15.134$ & $\begin{array}{l}\text { extubation - POD } 3 \\
\text { ICU discharge - POD } 6 \\
\text { hospital discharge - } \\
\text { POD } 12\end{array}$ \\
\hline 4 & $\begin{array}{l}F / 70 \\
29.3\end{array}$ & STEMI & CABG x 3 & $\mathrm{AH}$ & $\begin{array}{l}3 \text { boluses }+0.1-0.15 \\
\mu \mathrm{g} / \mathrm{kg} / \min (24 \mathrm{~h})\end{array}$ & $\begin{array}{l}\text { CI } 2.4 \text { / } 2.9 \\
\text { SVR } 1123 \text { / } 972 \\
\text { LVEF 46\% / 54\% }\end{array}$ & $>50 / 22.864$ & $\begin{array}{l}\text { extubation - POD } 1 \\
\text { ICU discharge - POD } 2 \\
\text { hospital discharge - } \\
\text { POD } 7\end{array}$ \\
\hline 5 & $\begin{array}{l}\text { F / } 57 \\
24.9\end{array}$ & STEMI & CABG x 3 & $\begin{array}{l}\mathrm{AH}, \mathrm{DM}, \mathrm{IABP} \\
\text { on POD } 1\end{array}$ & $\begin{array}{l}3 \text { boluses }+0.1-0.15 \\
\mu \mathrm{g} / \mathrm{kg} / \mathrm{min}(48 \mathrm{~h})\end{array}$ & $\begin{array}{l}\text { CI } 2.3 \text { / } 2.9 \\
\text { SVR } 1738 \text { / } 1264 \\
\text { LVEF 36\% / 48\% }\end{array}$ & $>50 / 20.416$ & $\begin{array}{l}\text { extubation - POD } 2 \\
\text { ICU discharge - POD } 5 \\
\text { hospital discharge - } \\
\text { POD } 11\end{array}$ \\
\hline 6 & $\begin{array}{l}\text { M / } 69 \\
29.4\end{array}$ & NSTEMI & CABG x 2 & $\begin{array}{l}\text { previous } 2 \mathrm{MI}+ \\
\text { CABG x } 4, \\
\text { postoperative } \\
\text { delirium }\end{array}$ & $\begin{array}{l}2 \text { boluses }+0.1-0.15 \\
\mu \mathrm{g} / \mathrm{kg} / \mathrm{min} \text { for } 48 \mathrm{~h}\end{array}$ & $\begin{array}{l}\text { CI } 2.7 \text { / } 3.2 \\
\text { SVR } 970 \text { / } 687 \\
\text { LVEF 36\% / 45\% }\end{array}$ & $0.878 / 0.212$ & $\begin{array}{l}\text { extubation - POD } 1 \\
\text { ICU discharge - POD } 5 \\
\text { hospital discharge - } \\
\text { POD } 11\end{array}$ \\
\hline 7 & $\begin{array}{l}M / 63 \\
25.1\end{array}$ & unstable AP & $\begin{array}{l}\text { OPCAB } \times 3 \\
\text { TEA }\end{array}$ & $\begin{array}{l}\text { recent NSTEMI, } \\
\mathrm{AH}, \mathrm{DM} \text {, } \\
\text { left } \mathrm{ACC} / \mathrm{ACI} / \\
\mathrm{ACE} \text { stenosis }\end{array}$ & $\begin{array}{l}3 \text { boluses }+0.1-0.15 \\
\mu \mathrm{g} / \mathrm{kg} / \mathrm{min}(24 \mathrm{~h})\end{array}$ & $\begin{array}{l}\text { CI } 1.9 \text { / } 3.5 \\
\text { SVR } 1299 \text { / } 679 \\
\text { LVEF 22\% / 45\% }\end{array}$ & $1.180 / 0.793$ & $\begin{array}{l}\text { extubation - POD } 1 \\
\text { ICU discharge - POD } 2 \\
\text { hospital discharge - } \\
\text { POD } 7\end{array}$ \\
\hline 8 & $\begin{array}{l}M / 70 \\
25.5\end{array}$ & unstable AP & OPCAB x 3 & $\mathrm{AF}$ & $\begin{array}{l}3 \text { boluses }+0.1-0.15 \\
\mu \mathrm{g} / \mathrm{kg} / \min (36 \mathrm{~h})\end{array}$ & $\begin{array}{l}\text { CI } 2.2 \text { / } 3.1 \\
\text { SVR } 1043 \text { / } 748 \\
\text { LVEF 27\% / 39\% }\end{array}$ & $1.403 / 0.644$ & $\begin{array}{l}\text { extubation - POD } 1 \\
\text { ICU discharge - POD } 2 \\
\text { hospital discharge - } \\
\text { POD } 9\end{array}$ \\
\hline 9 & $\begin{array}{l}\mathrm{M} / 80 \\
25.4\end{array}$ & unstable AP & OPCAB $\times 2$ & $\begin{array}{l}\mathrm{AH}, \mathrm{DM}, \mathrm{COPB} \\
\text { respiratory } \\
\text { insufficiency }\end{array}$ & $\begin{array}{l}2 \text { boluses }+0.1-0.2 \mu \mathrm{g} \\
\mathrm{kg} / \mathrm{min}(24 \mathrm{~h})\end{array}$ & $\begin{array}{l}\text { /CI } 2.0 \text { / } 2.4 \\
\text { SVR } 1060 \text { / } 846 \\
\text { LVEF 55\% / 63\% }\end{array}$ & $3.081 / 0.626$ & $\begin{array}{l}\text { extubation - POD } 1 \\
\text { ICU discharge - POD } 8 \\
\text { hospital discharge - } \\
\text { POD } 20\end{array}$ \\
\hline 10 & $\begin{array}{l}\mathrm{F} / 66 \\
25.2\end{array}$ & unstable AP & CABG $\times 2$ & $\begin{array}{l}\text { AH, hypothy- } \\
\text { roidism }\end{array}$ & $\begin{array}{l}2 \text { boluses }+0.1-0.2 \mu \mathrm{g} \\
\mathrm{kg} / \mathrm{min}(24 \mathrm{~h})\end{array}$ & $\begin{array}{l}\text { /CI } 1.6 \text { / } 3.4 \\
\text { SVR } 1292 \text { / } 1071 \\
\text { LVEF 47\% / 60\% }\end{array}$ & $1.691 / 0.426$ & $\begin{array}{l}\text { extubation - POD } 1 \\
\text { ICU discharge - POD } 4 \\
\text { hospital discharge - } \\
\text { POD } 9\end{array}$ \\
\hline
\end{tabular}

ACC/ACE/ACI , common/external/internal carotid artery; ACS, acute coronary syndromes; AF, atrial fibrillation; AH, arterial hypertension; AP, angina pectoris; BMI, body mass index; CABG, coronary artery bypass grafting ("on-pump" type of surgery); CI, cardiac index (ml•m-2); COPB, chronic obstructive pulmonary disease; CRRT, continuous renal replacement therapy; DM, diabetes mellitus; F, female; HD, hemodialysis; IABP, intra-aortic balloon pump; IC, intracoronary; ICU, intensive care unit; LVEF, left ventricular ejection fraction; M, male; MI, myocardial infarction; NSTEMI, non-ST segment elevation myocardial infarction; OPCAB, "off-pump" coronary artery bypass (type of surgery); POD, postoperative day; STEMI, ST segment elevation myocardial infarction; SVR, systemic vascular resistance (dynes•sec $\bullet$ cm-5); TEA, thrombendarterectomy. 
Table 2. Preoperative vs. immediate postoperative values (or ICU discharge values for troponin I) of CI, SVR, LVEF, and troponin I variables in all patients (i.e. before and after IC administration of levosimendan).

\begin{tabular}{llll}
\hline variables & preoperative MED (IQR) & postoperative MED (IQR) & $\mathbf{P}^{*}$ \\
\hline $\mathrm{CI}(\mathrm{ml} \cdot \mathrm{m}-2)$ & $2.2(1.9-2.5)$ & $3.1(2.9-3.4)$ & 0.005 \\
\hline SVR $($ dynes $\bullet$ sec $\bullet \mathrm{cm}-5)$ & $1173.0(1062.7-1278.2)$ & $882.5(763.5-993.0)$ & 0.005 \\
\hline LVEF $(\%)$ & $44.5(36.0-46.7)$ & $53.5(45.7-59.2)$ & 0.005 \\
\hline troponin I (ng•ml-1) & $26.54(1.48-50)$ & $3.92(0.63-18.37)$ & 0.005 \\
\hline
\end{tabular}

CI, cardiac index; IQR, interquartile range; LVEF, left ventricular ejection fraction; MED, median; SVR, systemic vascular resistance.

${ }^{*} \mathrm{P}$ - value derived from Wilcoxon signed-ranks test; values presented as MED (IQR).

\section{REFERENCES}

1. Tritapepe L, De Santis V, Vitale D, Guarracino F, Pellegrini F, Pietropaoli P, et al. Levosimendan pre-treatment improves outcomes in patients undergoing coronary artery bypass graft surgery. Br J Anaesth 2009;102(2):198-204.

2. Parissis JT, Rafouli-Stergiou P, Stasinos V, Psarogiannakopoulos P, Mebazaa A. Inotropes in cardiac patients: update 2011. Curr Opin Crit Care 2010;16(5):432-41.

3. Mebazaa A, Pitsis AA, Rudiger A, Toller W, Longrois D, Ricksten SE, et al. Clinical review: Practical recommendations on the management of perioperative heart failure in cardiac surgery. Crit Care 2010;14(2):201.

4. Toller W, Heringlake M, Guarracino F, Algotsson L, Alvarez J, Argyriadou H, et al. Preoperative and perioperative use of levosimendan in cardiac surgery: European expert opinion. Int J Cardiol 2015;184:323-36.

5. Pieske B. Levosimendan in regional myocardial ischemia. Cardiovasc Drugs Ther 2002;16(5):379-81.

6. Caimmi PP, Molinari C, Uberti F, Micalizzi E, Valente G, Mary DA, et al. Intracoronary levosimendan prevents myocardial ischemic damages and activates survival signaling through ATP-sensitive potassium channel and nitric oxide. Eur J Cardiothorac Surg 2011;39(4):e59-67.

7. Malmberg M, Vähäsilta T, Saraste A, Koskenvuo JW, Pärkkä JP, Leino K, et al. Intracoronary levosimendan during ischemia prevents myocardial apoptosis. Front Physiol 2012;3:17.

8. Caimmi PP, Kapetanakis EI, Beggino C, Molinari C, Giustini G, Crosio E, et al. Management of acute cardiac failure by intracoronary administration of levosimendan. J Cardiovasc Pharmacol 2011;58(3):246-53.

9. Givertz MM, Andreou C, Conrad CH, Colucci WS. Direct myocardial effects of levosimendan in humans with left ventricular dysfunction: alteration of force-frequency and relaxation-frequency relationships. Circulation 2007;115(10):1218-24.

10. Jamali IN, Kersten JR, Pagel PS, Hettrick DA, Warltier DC. Intracoronary levosimendan enhances contractile function of stunned myocardium. Anesth Analg 1997;85(1):23-9. 